\title{
Successful stenting of a life threatening pulmonary arterial stenosis
}

\author{
David Mulcahy, Ulrich Sigwart, Jane Somerville
}

\begin{abstract}
A 35 year old woman with multiple pulmonary arterial stenoses and occlusions was admitted with increasingly severe breathlessness. Balloon angioplasty of a life threatening stenosis in a remaining pulmonary artery branch was attempted on two occasions but without success. When a self-expanding stainless steel stent was inserted at the site of stenosis after $a$ further angioplasty acute localised pulmonary oedema developed as blood flow distal to the lesion increased considerably. This was followed by a slow and dramatic improvement in the patient's condition. She no longer required an urgent transplant and resumed an active life.
\end{abstract}

Little is known about the long term natural history and late complications of multiple pulmonary arterial stenoses; progressive occlusion of such stenoses, however, causes increasing hypoxia and breathlessness. Lately, balloon angioplasty has been used to dilate stenosed pulmonary arterial branches, ${ }^{1}$ and in animals stents have been used to prevent restenosis in such lesions. ${ }^{2}$ We report a patient with multiple pulmonary arterial stenoses and occlusions who underwent stent insertion at the site of a critical stenosis in a remaining pulmonary arterial branch after two failed balloon angioplasties.

\section{Case report}

A 35 year old female patient of JS was admitted for investigation of an 18 month history of increasing breathlessness on exertion that was severely limiting her quality of life (ability index 4). She also reported profound tiredness and an increasing susceptibility to respiratory tract infections and chronic cough.

A murmur had been noted when she was four. When she was five a patent ductus arteriosus, an atrial septal defect, and pulmonary hypertension were found on investigation. The ductus was closed surgically. Pulmonary angiography was performed because a continuous murmur persisted. It showed multiple bilateral pulmonary arterial stenoses. The patient remained well until 1973 (age 13) when further investigations were prompted by the development of cyanosis after exertion. Pulmonary angiography showed a grossly dilated main pulmonary artery (pressure $86 / 40 \mathrm{~mm}$ $\mathrm{Hg}$ ) with multiple areas of stenosis or occlusion in the branches of both pulmonary arteries. There was no evidence of shunting in either direction across the atrial septal defect. The comment was made that "the vast majority of the cardiac output passed through the left lower lobe pulmonary artery". She remained well with a moderate exercise capacity (ability index 2), coping well with the delivery of a live child by caesarean section at 35 weeks' gestation in 1984. She required treatment on two occasions for suspected but unproven pulmonary tuberculosis. She presented in 1990 (after being lost to follow up for the previous four years) with increasing breathlessness over the preceding 18 months. During an admission to assess and treat her increasingly debilitating chronic cough, an exercise test, performed with continuous oximetric readings, showed that after 3.5 minutes of slow walking the arterial saturation fell from $86 \%$ at heart rate 72 beats/ $\min$ to $54 \%$ with heart rate 94 beats $/ \mathrm{min}$. In view of her deteriorating state she was readmitted for further investigation.

Examination showed her to be small in stature with moderate clubbing and with cyanosis at rest. The pulse was 70 beats $/ \mathrm{min}$ and regular. Blood pressure was $110 / 70 \mathrm{~mm} \mathrm{Hg}$. There was a giant ' $a$ ' wave in the jugular venous pulse. The right ventricle and pulmonary artery were palpable with a loud pulmonary component to the second heart sound and ejection systolic and an early diastolic murmur in the pulmonary area. There was a loud continuous murmur over the left posterior region of the chest.

Relevant investigations included haemoglobin $(156 \mathrm{~g} / \mathrm{l})$ and packed cell volume (48 $1 / 1)$. The chest $x$ ray was abnormal with nodular shadowing in the mid and lower zones of the left lung. The right lung was reduced in volume with cavities, cyst formation, and pleural thickening in the right mid and upper zones and linear shadowing in all areas of the lung. The pulmonary conus was prominent and the pulmonary arteries much attenuated. The heart was large (cardiothoracic ratio 13/24.5). Resting electrocardiography showed sinus rhythm, first degree heart block, right axis deviation, and right ventricular hypertrophy.

Cardiac catheterisation confirmed right to 
left shunting across the atrial septal defect (saturations: left upper lobe pulmonary vein $98 \%$, left atrium $93 \%$, aorta $88 \%$ ). The main pulmonary artery pressure was $120 / 35 \mathrm{~mm} \mathrm{Hg}$ (mean $65 \mathrm{~mm} \mathrm{Hg}$ ) and left lower lobe pulmonary artery pressure was $25 / 15 \mathrm{~mm} \mathrm{Hg}$ (mean $20 \mathrm{~mm} \mathrm{Hg}$ ). Pulmonary angiography showed a blocked right pulmonary artery and multiple stenoses and occlusions of the left pulmonary artery branches with a significant stenosis of the left lower lobe pulmonary arterial branch (fig 1). The main pulmonary artery was aneurysmal. The right lung filled by acquired multiple collateral blood vessels from the bronchial arteries, the left circumflex artery, and the right internal mammary artery. The left lung was also supplied by abnormal collaterals from the left internal mammary artery. All the collaterals were small.

In view of the importance of the persisting pulmonary arterial supply to the left lower lobe we planned to perform an elective balloon dilatation of the left lower lobe pulmonary arterial stenosis. The patient deteriorated, however, and was re-admitted as an emergency with severe breathlessness, dizziness, and chest pain, which we believed might have been caused by "silting up" of one or more remaining pulmonary arterial branches. An infusion of streptokinase was started and she was referred for urgent heart-lung transplantation. When the chest pain became worse the streptokinase infusion was replaced with a heparin infusion, and the patient gradually stabilised, with variable oxygen saturations from $60 \%$ to $85 \%$. Because she showed slight clinical improvement, we considered that it was worthwhile to attempt angioplasty of the lesion. She underwent balloon dilatation $(6 \mathrm{~mm}$ and $8 \mathrm{~mm}$ balloons) of the left lower lobe pulmonary arterial stenosis on two occasions over the next two weeks; however, there was little change in the transstenotic gradient and little overall improvement in clinical state. The stenotic area was noted to be "rubbery" in its response to stretch. The patient then underwent a third procedure with a $10 \mathrm{~mm}$ balloon dilatation of the lesion followed by insertion of a modified self-expanding $12 \mathrm{~mm}$ stainless steel wall stent device, and re-inflation of a $10 \mathrm{~mm}$ balloon within the stent to smooth it out (fig 2). Mean main pulmonary artery pressure fell from 70 $\mathrm{mm} \mathrm{Hg}$ to $55 \mathrm{~mm} \mathrm{Hg}$ and mean left lower lobe pulmonary artery pressure rose from $7 \mathrm{~mm} \mathrm{Hg}$ to $34 \mathrm{~mm} \mathrm{Hg}$ immediately after the procedure. There was no appreciable change in arterial saturation.

An hour later she became breathless with hypotension, bradycardia, and severe hypoxia. A chest radiograph showed evidence of pulmonary oedema localised to the left lower lobe. This resolved over the next five days, and the patient was discharged in a stable condition on anticoagulants and diuretics.

When she was reviewed at an outpatient clinic 10 months after stent insertion there had been a slow but appreciable improvement in exercise capacity. Informal exercise testing two months after discharge had shown only a small drop in oxygen saturation $(91 \%$ to $86 \%)$ at the end of exercise; she had started to swim again at this time. In view of the clinical improvement

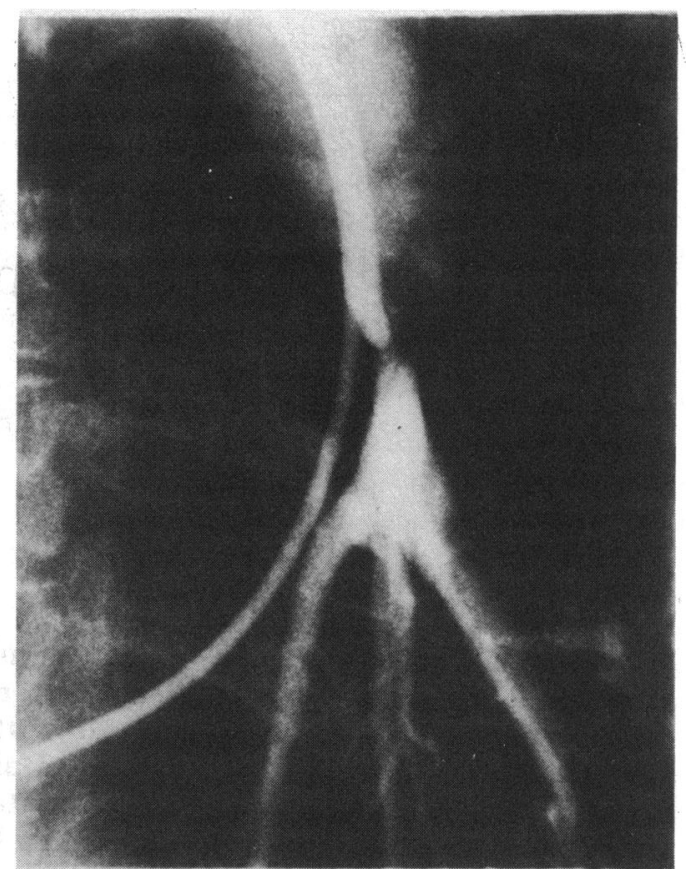

Figure 1 Angiographic demonstration of a severe stenosis in the left lower lobe pulmonary artery.

the patient was taken off the urgent transplant list. She attended the outpatient clinic to seek advice about another pregnancy, but was strongly advised against this.

\section{Discussion}

Various aspects of this case deserve comment. Though this patient was accepted on to the emergency heart-lung transplant waiting list the previous surgery and the abundance of abnormal collaterals supplying both lungs would have made her a high risk candidate and control of bleeding a major problem. In addition, surgery performed to directly relieve hypoplastic and stenotic branch pulmonary

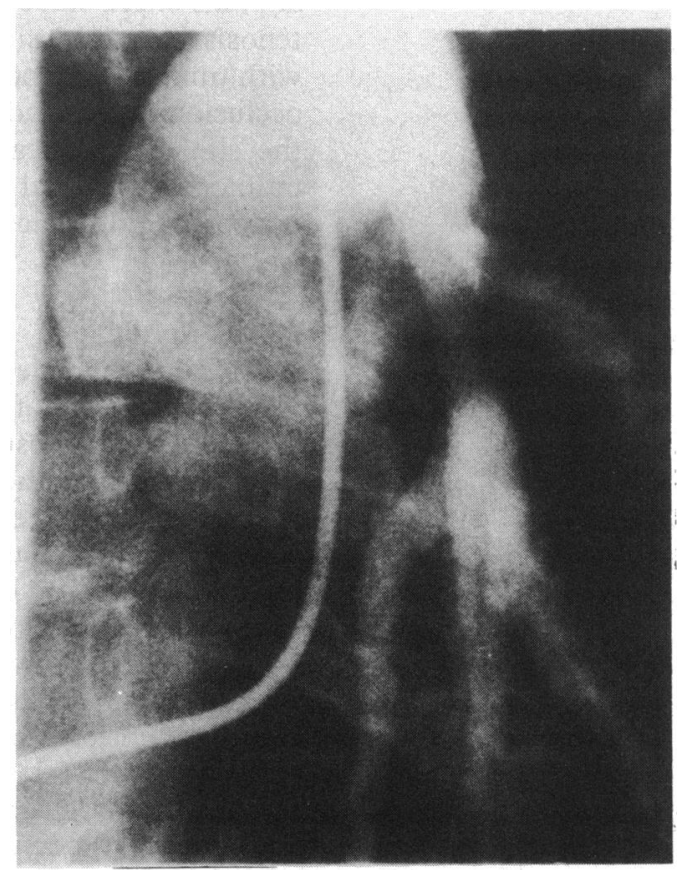

Figure 2 Angiographic demonstration of left lower lobe pulmonary artery after stent implantation. There was excellent flow through the prosthesis, and the distal branch vessels appeared to be larger. $A$ branch artery was seen to fill well from the side of the inserted stent. 
arteries has been reported to be difficult and often ineffective. ${ }^{34}$ Because of the complexity of the surgical approach, balloon angioplasty has become popular as a relatively non-invasive method of relieving obstruction to flow within the pulmonary circulation. This method has particular applications in the management of intraparenchymal pulmonary artery stenoses, which may be inaccessible to surgery. Balloon angioplasty has been shown to relieve pulmonary artery stenoses by tearing the vascular intima and media, allowing remodelling and healing at a larger diameter. ${ }^{5}$

Rothman and colleagues have recently published the results of 218 balloon angioplasty procedures in 135 patients with branch pulmonary artery stenoses. ${ }^{1}$ They reported a success rate of $60 \%$, a mortality rate of $1 \%$, and aneurysm formation in $3 \%$. Our patient is at the upper end of the series age, and it has been suggested that procedural success diminishes with the age of the patient, ${ }^{6}$ however, Rothman et al found no such relation. ${ }^{1} \mathrm{He}$ did find a positive correlation between success and balloon to artery size ratio. The first two attempts at dilatation in our patient probably failed because the balloon was too small, however, on the third occasion the balloon was large enough but it still did not produce lasting dilatation or improvement in pressure dynamics. Rothman et al reported that according to diameter criteria 25 of 57 procedures in those with follow up angiograms were not initially successful, suggesting a significant incidence where stenoses were not adequately dilated or where there was immediate recoil of such lesions. This was true of our patient, in whom, despite maximal inflation of the balloon without waisting, the stenosis rapidly returned to its pre-dilatation diameter.

Though coronary angioplasty is widely used in patients with coronary artery stenoses, restenosis rates remain high (up to $35 \%$ ). ${ }^{78}$ Because of this many investigators have attempted to design "mechanical barriers" or stents to insert at the site of lesions to prevent restenosis. ${ }^{910}$ In view of the precarious clinical condition of our patient, and the apparent lack of success with conventional balloon dilatation, a modified stainless steel self-expanding mesh stent was inserted in the branch pulmonary artery after dilatation of the lesion. Further dilatations from within the expanded prosthesis were used to smooth out the stent. The beneficial effects were immediately obvious with a striking increase in distal pressures and reduction in main pulmonary artery pressures. The improvement in flow through the stented lesion, however, caused acute localised pulmonary oedema or "flooding", a recognised complication after successful balloon dilatation of branch pulmonary artery stenoses. ${ }^{111}$ Wood Arnold and colleagues noted that after dilatation both relief of stenosis and the distal mean pulmonary artery pressure were both greater in patients with this complication than in those without. ${ }^{11}$ They also noted that the degree of change in distal pulmonary artery pressure was crucial to the development of oedema, concluding that an increase of $>70 \%$ in vessel diameter, an increase of $>170 \%$ in distal pressure, and a distal pressure of $>20 \mathrm{~mm} \mathrm{Hg}$ immediately after dilatation identified those at risk for the development of transient localised pulmonary oedema. In our patient mean distal pulmonary artery pressure increased considerably after stent insertion, not surprisingly this led to acute pulmonary oedema. If the use of stents in the management of branch pulmonary artery stenoses becomes more widespread, this complication is likely to become more common. Such procedures should ideally be covered by prophylactic oxygen and intravenous diuretics. It is reassuring to know that standard management of pulmonary oedema leads to gradual resolution of the problem and that the distal pulmonary artery vasculature can rapidly accommodate higher flow and pressure even when hypoperfusion has developed gradually over several decades.

O'Loughlin and colleagues studied the practicality of stent insertion in pulmonary arteries in dogs, and noted that early stent migration occurred but was overcome by the use of larger balloons. ${ }^{2}$ They noted that side branches crossed by the stents remained patent. Pathological evaluation showed that struts that were in apposition to the vessel wall had a smooth neoendothelial covering. No thromboses or distal thromboemboli were found. As with coronary stents in humans, however, adequate anticoagulation is mandatory at the time of and after stent insertion, and although neoendothelialisation seems to occur in a matter of weeks, most workers would recommend anticoagulation for 3-6 months in view of the thrombotic tendency resulting from stent insertion. In view of the raised pulmonary artery pressure, anticoagulation for life might be the more sensible approach in those with intrapulmonary stents.

Although we cannot be certain of the long term outcome in our patient, the short term palliation of symptoms was strikingly successful. Pulmonary artery stenting seems to be both feasible and effective. With technical advances and measures to counter the complications of thrombosis and "flooding", the stent may increasingly be used in patients with pulmonary artery stenosis.

1 Rothman A, Perry SB, Keane JF, Lock JE. Early results and follow-up of balloon angioplasty for branch pulmonar artery stenoses. J Am Coll Cardiol 1990;15:1109-17.

2 O'Loughlin MP, Myers TJ, Kearney DL, Mullins CE Balloon-expandable stents in pulmonary arteries and systemic veins-long-term experimental follow-up [abstract]. J Am Coll Cardiol 1991;82:655.

3 McGoon DC, Kincaid OW. Stenosis of branches of the pulmonary artery: surgical repair. Med Clin North Am 1964;48:1083-8.

4 Cohn LH, Sanders JH, Collins JJ. Surgical treatment of congenital unilateral pulmonary arterial stenosis with contralateral pulmonary hypertension. Am J Cardio 1976;38:257-60.

5 Lock JE, Niemi T, Einzig S, Amplatz K, Burke B, Bass JL. Transvenous angioplasty of experimental branch pulmonary artery stenosis in newborn lambs. Circulation $1981 ; 64: 886-92$.

6 Ring JC, Bass JL, Marvin W, et al. Management of congenital stenosis of a branch pulmonary artery with balloon dilation angioplasty. $J$ Thorac Cardiovasc Sur 1985;90:35-44.

7 Leimgrubber PP, Roubin GS, Hollman J, et al. Restenosis after successful coronary angioplasty in patients with after successful coronary angioplasty in patient

8 McBride W, Lange RA, Hillis LD. Restenosis after successful cong ful coronary angioplasty. $N$ Engl $J$ Med 1988;318:1734-7.

9 Sigwart U, Puel J, Mirkowitch V, Joffre F, Kappenberger L. Intravascular stents to prevent occlusion and restenosis after tran.

10 Schatz RA. A view of vascular stents. Circulation Schatz RA. A

Wood Arnold L, Keane JF, Kan JS, Fellows KE, Lock JE. Transient unilateral pulmonary edema after successful transient unilateral pulmonary edema artery stenosis. Am J Cardiol 1988;62:327-30. 